\title{
Transient asymptomatic hepatitis B surface antigenemia following recombinant Recombivax $B$ hepatitis $B$ vaccine in a 42 -year-old ESRD patient on maintenance hemodialysis
}

\author{
Macaulay Amechi Chukwukadibia Onuigbo ${ }^{1,3^{*}}$, Catherine Kress ${ }^{4}$, Carol Webb ${ }^{3}$, Nneoma Agbasi ${ }^{2}$ \\ ${ }^{1}$ Mayo Clinic College of Medicine, Rochester, MN, USA \\ ${ }^{2}$ North East London NHS Foundation Trust, UK \\ ${ }^{3}$ Department of Nephrology, Mayo Clinic Health System, Eau Claire, WI, USA \\ ${ }^{4}$ Des Moines University, D.O. Class of 2018, USA
}

\section{A R T I C L E I N F 0}

Article Type:

Case Report

\section{Article History:}

Received: 8 September 2017

Accepted: 24 November 2017

ePublished: 14 December 2017

\section{Keywords:}

End-stage renal disease

Engerix B vaccine

HbSAgenemia

Hemodialysis

Hepatitis B

Recombivax B vaccine

Vaccination

\begin{abstract}
A B S T R A C T
A 42-year old Caucasian end-stage renal disease (ESRD) male patient with past medical history including diabetes, hypertension, pulmonary hypertension (RVSP of $53 \mathrm{~mm} \mathrm{Hg}$ ) and tobacco use tested positive for hepatitis B surface antigen (HBsAg) after a routine blood screening test using an enzyme immunoassay (EIA) method. He however was otherwise asymptomatic for any symptoms of hepatitis. On further review, we confirmed that he had received an intramuscular dose of Recombivax (Merck) vaccine, $1 \mathrm{~mL}=40 \mu \mathrm{g}$, 5 days previously. Follow up liver panel test was unremarkable with normal bilirubin and transaminases. Repeat serology testing at Mayo Clinic, Rochester the next day for a confirmatory neutralizing antibody assay test was negative for HBsAg. Furthermore, hepatitis B core IgM antibody, hepatitis B surface antibody, hepatitis B surface antibody qualitative, hepatitis B surface antibody quantitative test $<0.1(<8.0 \mathrm{mIU} / \mathrm{mL})$, hepatitis B e antibody and hepatitis B core total antibody tests all subsequently returned negative. This is the second case of transient postvaccination HbSAgenemia observed in our hemodialysis Unit in Northwestern Wisconsin in the last 7 years. Once again, as we posited in our 2010 report, we reemphasize previous recommendations that patients who receive hepatitis $B$ vaccinations should not be screened for HBsAg less than 4 weeks following a hepatitis B vaccination.
\end{abstract}

\section{Implication for health policy/practice/research/medical education:}

We report the rare phenomenon of transient hepatitis B surface antigenemia (HbsAgenemia) following preventative hepatitis $B$ vaccination with Recombivax B vaccine. A misdiagnosis of a hepatitis B infection could lead to potentially harmful albeit unnecessary laboratory work up, unwarranted psychological stressors to the patient and family, and a feeling of, anxiety and isolation. We reemphasize previous recommendations that patients who receive hepatitis B vaccinations should not be screened for HBsAg less than 4 weeks following a hepatitis B vaccination.

Please cite this paper as: Onuigbo MAC, Kress C, Webb C, Agbasi N. Transient asymptomatic hepatitis B surface antigenemia following recombinant Recombivax B hepatitis B vaccine in a 42-year-old ESRD patient on maintenance hemodialysis. J Nephropharmacol. 2017;7(1):43-45. DOI: 10.15171/npj.2018.10.

\section{Introduction}

Hemodialysis exposes end-stage renal disease (ESRD) patients to significantly higher risks for hepatitis B virus (HBV) infection, a major public health scourge (1-4). Current US CDC guidelines and recommendations, last comprehensively revised in 2001, followed in US hemodialysis units, and simultaneously adhered to worldwide, among other recommendations, require monthly screening hepatitis B surface antigen (HBsAg) testing in all hemodialysis patients together with mandatory $\mathrm{HBV}$ vaccination of all hemodialysis patients (5-7). Furthermore, the usual hepatitis B vaccination protocol calls for a series of three recombinant $\mathrm{HbSAg}$ vaccines given intramuscularly at 0,1 and 6 months respectively. In 2010, we reported in the journal Renal Failure, the rare phenomenon of transient HbSAgenemia two days following Engerix B (GlaxoSmithKline) vaccination in an 81-year old Caucasian woman on 
maintenance in-center outpatient hemodialysis (8). We just recently encountered a similar event, this time 5 days after Recombivax B (Merck) vaccination.

\section{Case Presentation}

A 42-year-old Caucasian male patient, on maintenance hemodialysis for end stage renal disease since December 2016 was admitted to the Hospitalist Service and underwent an elective placement of a left upper arm basilic vein transposition arteriovenous fistula on June 29, 2017. The patient has a history including diabetes, hypertension, pulmonary hypertension (RVSP of $53 \mathrm{~mm} \mathrm{Hg}$ ), smoker who started dialysis December 2016 via a tunnelled right internal jugular vein dialysis catheter for symptomatic diabetic nephropathy for proteinuria ESRD, with anasarca and metabolic acidosis and had transferred to our unit in mid-March 2017. He had subsequently undergone a cardiac catheterization, elective in early June 2, 2017with reported placement of a stent in the LAD. The AV fistula transposition was completed successfully on June 29, 2017 and he was soon confirmed to have a left foot non-healing osteomyelitis complicating peripheral arterial disease. Despite an antecedent left lower extremity angiogram with angioplasty and stenting carried out on July 1, 2017, he still ended up requiring a left lower extremity below knee amputation on July 6, 2017. In addition, a dark and painful necrotizing lesion involving the tip of the penis was reported and was suspected to represent some form of calciphylaxis. His calcium-based phosphate binder was discontinued and replaced with Sevelamer and he was then empirically started on intravenous sodium thiosulfate. He was discharged from the hospital to a Swing bed facility on July 12, 2017.

The next day, on July 13, in an outpatient dialysis unit, we were notified that he had tested positive for HBsAg after a routine blood screening test. He however was otherwise asymptomatic for symptoms of hepatitis. At the time of this notification, we were not immediately aware of the recent hepatitis B vaccination with Recombivax (Merck) vaccination that was given as an intramuscular injection $(1 \mathrm{~mL}=40 \mu \mathrm{g})$ on July 8, 2017.

The following day, on July 14 a repeat blood sample was sent to Mayo Clinic, Rochester, for a confirmatory neutralizing antibody assay test which subsequently returned negative for HBsAg. The following additional hepatitis B-related tests all returned negative; hepatitis B core IgM antibody, hepatitis B surface antibody, hepatitis B surface antibody qualitative, hepatitis B surface antibody quantitative test $<0.1(<8.0 \mathrm{mIU} / \mathrm{mL})$, hepatitis $\mathrm{B}$ e antibody and hepatitis $\mathrm{B}$ core total antibody. In the interim, however, as a precautionary measure, he was dialyzed on a designated "other" hemodialysis machine. Further testing on July 17 th revealed normal liver function test results for ALT $5 \mathrm{U} / \mathrm{L}$ (7-55 U/L), AST $14 \mathrm{U} / \mathrm{L}$ (8-48 U/L), total bilirubin $0.3 \mathrm{mg} / \mathrm{dL}(<1.2 \mathrm{mg} / \mathrm{dL})$, and direct bilirubin $<0.2 \mathrm{mg} /$ $\mathrm{dL}(0.0-0.3 \mathrm{mg} / \mathrm{dL})$. The elevated alkaline phosphatase $179 \mathrm{U} / \mathrm{L}(45-115 \mathrm{U} / \mathrm{L})$ was not a differentiated test to separate hepatic versus other isoenzymes of alkaline phosphatase, most likely from bone source secondary to renal osteodystrophic changes. Patient does not have a history of any blood transfusions.

On subsequent review, it became clear that the patient had received the first dose of the Recombivax vaccination on 1-7-17, the second dose, one month later, on 2-9-17 and the third index vaccination, 6 months later on 7-817 , according to established protocol. In our dialysis units, routine blood screening for $\mathrm{HbSAg}$ is done locally with an enzyme immunoassay (EIA) method-GS HBsAg EIA 3.0 assay kit manufactured by Bio-Rad Laboratories (Redmond, WA, USA; US License No. 1109) (9). This is a state of the art third generation mouse monoclonal antibody-based EIA. However, any positive HBsAg tests are then confirmed by a neutralizing antibody assay, carried out at Mayo Clinic, Rochester (9). Of note, he had previously tested HbSAg negative in March 2017, in May 2017 and last in June 2017.

\section{Discussion}

Once again, we have demonstrated transient false positive HbSAgenemia in an ESRD patient, this time, five days following Hepatitis $\mathrm{B}$ vaccination with Recombivax vaccine. In 2010, we reported a similar rare finding in an 81-year old ESRD female patient, two days following Engerix B vaccine (8).

The first cases of transient hepatitis B surface antigenemia (HBsAg) in adults following HBV immunization were reported in the 1990s (10-12). Ly et al, who demonstrated transient HBsAg in eight out of nine hemodialysis patients following $\mathrm{HBV}$ vaccine concluded that hemodialysis patients should not be screened for HBsAg within a week of $\mathrm{HBV}$ vaccination and that positive $\mathrm{HBsAg}$ within a month of hepatitis B vaccination must be interpreted with caution (13). To our knowledge, by 2009, all previous reports of transient positive HBsAg test results following Hepatitis B vaccination had followed Engerix B administration (10-14). Furthermore, our 2010 report also followed an Engerix B vaccine (8). In this report, the patient had received his third Recombivax $B$ vaccine instead. Notably, both Engerix B and Recombivax B vaccines are both recombinant antigen derived vaccines that have no association with human blood or blood products. It remains unclear why the preponderance of reports of transient post-vaccination HbSAgenemia have followed the Engerix B vaccine (8-14).

Once again, as we posited in our 2010 report, we would want to reemphasize previous recommendations that patients who receive hepatitis $B$ vaccinations should not be screened for HBsAg less than 4 weeks following a Hepatitis B vaccination $(8,9,13)$. The longest reported interval between an Engerix B vaccination and a positive HbSAg test was eighteen days in a 17-year old potential American male blood donor (15). A misdiagnosis of a hepatitis B infection could lead to potentially harmful albeit unnecessary laboratory work up, unwarranted psychological stressors to the patient and family, and a feeling of anxiety and isolation for the patient as he will 
no longer be allowed to dialyze anywhere else in the dialysis unit except in the "dedicated" dialysis machines $(8,9,15)$. Moreover, at least theoretically, this misdiagnosis for a hemodialysis patient has the potential to increase the chances of the real vertical transmission of Hepatitis $\mathrm{B}$ to the patient from a truly hepatitis $\mathrm{B}$ patient by being dialyzed on the same "dedicated" machine (1-5).

\section{Conclusion}

We report the rare phenomenon of transient hepatitis B surface antigenemia (HbSAg) following preventative hepatitis B vaccination with Recombivax B vaccine. A misdiagnosis of a hepatitis B infection could lead to potentially harmful albeit unnecessary laboratory work up, unwarranted psychological stressors to the patient and family, and a feeling of, anxiety and isolation. We reemphasize previous recommendations that patients who receive Hepatitis $B$ vaccinations should not be screened for HBsAg less than 4 weeks following a Hepatitis B vaccination.

\section{Authors' contribution}

MACO: Conception, design, acquisition of data, data analysis, interpretation of data, literature review, drafting the article and final approval of manuscript. NA: Critical revising for important intellectual content, design, final approval of manuscript. CK: Acquisition of data and final approval of manuscript. CW: Acquisition of data and final approval of manuscript.

\section{Conflicts of interest}

The authors declare no conflicts of interest. The authors alone are responsible for the content and writing of the article.

\section{Ethical considerations}

Ethical issues (including plagiarism, data fabrication, double publication) have been completely observed by the authors. The patient has given his informed consent regarding this case report.

\section{Funding/Support}

None.

\section{References}

1. Garibaldi RA, Forrest JN, Bryan JA, Hanson BF, Dismukes WE. Hemodialysis-associated hepatitis. JAMA. 1973;225:384-9.
2. London WT, Di Figlia M, Sutnick A, Blumberg BS. An epidemic of hepatitis in a chronic-hemodialysis unit. Australia antigen and differences in host response. $\mathrm{N}$ Engl J Med. 1969;281:571-8.

3. Cossart YE, Gillespie EH, Jones DM, Kelsey JC. Infection risks of haemodialysis-some preventive aspects. A report to the Public Health Laboratory Service by the Working Party on Haemodialysis Units. Br Med J. 1968; 3:454-60.

4. Girndt $M$, Köhler $H$. Hepatitis B virus infection in hemodialysis patients. Semin Nephrol. 2002;22:340-50.

5. Centers for Disease Control and Prevention (CDC). Recommendations for preventing transmission of infections among chronic hemodialysis patients. MMWR Recomm Rep. 2001;50:1-43.

6. Centers for Disease Control and Prevention (CDC). Viral Hepatitis Scientific Information-Specific Settings. https:// www.cdc.gov/hepatitis/populations/index.htm.

7. Centers for Disease Control and Prevention (CDC). 2006 Guidelines for Vaccinating Kidney Dialysis Patients and Patients with Chronic Kidney Disease. http://www.cdc. gov/vaccines/pubs/downloads/b_dialysis_guide-508.pdf. Accessed September 24, 2010. Published 2006.

8. Onuigbo MA, Nesbit A, Weisenbeck J, Hurlburt J. Hepatitis B surface antigenemia following recombinant Engerix B hepatitis B vaccine in an 81-year-old ESRD patient on hemodialysis. Ren Fail. 2010;32:531-2. doi: 10.3109/08860221003637369.

9. Onuigbo MA, Onuigbo NT. De novo HBV infection in a Mayo Clinic hemodialysis population: economic impact of reduced HBV testing and a call for changes in current US CDC guidelines on $\mathrm{HBV}$ testing protocols. Hemodial Int. 2012;1:32-8. doi: 10.1111/j.1542-4758.2012.00748.x.

10. Kloster B, Kramer R, Eastlund T, Grossman B, Zarvan B. Hepatitis B surface antigenemia in blood donors following vaccination. Transfusion. 1995;35:475-77.

11. Kear TM, Wright LS. Transient hepatitis B antigenemia in hemodialysis patients following hepatitis B vaccination. ANNA J. 1996;23:331-37.

12. Janzen L, Minuk GY, Fast M, Bernstein KN. Vaccineinduced hepatitis B surface antigen positivity in adult hemodialysis patients: Incidental and surveillance data. J Am Soc Nephrol. 1996;7:1228-34.

13. Ly D, Yee HF Jr, Brezina M, Martin P, Gitnick G, Saab S. Hepatitis B surface antigenemia in chronic hemodialysis patients: Effect of hepatitis B immunization. Am J Gastroenterol. 2002;97:138-141.

14. Fleischer L, Syed SS. Hepatitis B surface antigenemia in a neonate following vaccination with Pediarix. Clin Pediatr (Phila). 2009;48:311-12.

15. Lunn ER, Hoggarth BJ, Cook WJ. Prolonged hepatitis B surface antigenemia after vaccination. Pediatrics. 2000;105:E81.

Copyright $\odot 2018$ The Author(s); Published by Society of Diabetic Nephropathy Prevention. This is an open-access article distributed under the terms of the Creative Commons Attribution License (http://creativecommons.org/licenses/by/4.0), which permits unrestricted use, distribution, and reproduction in any medium, provided the original work is properly cited. 